\title{
SMARTCAT E WORDFAST ANYWHERE: SISTEMAS DE MEMÓRIAS DE TRADUÇÃO E A DOCUMENTAÇÃO NAÁREẢ AGRÍCOLA EXPORTAD̉ORA DE LIMÃO
}

\author{
SMARTCAT AND WORDFAST ANYWHERE: TRANSLATION \\ MEMORY SYSTEMS AND DOCUMENTATION ABOUT THE \\ LEMON EXPORT AGRICULTURAL AREA
}

Talita SERPA ${ }^{1}$

Ione Marina de LIMA $^{2}$

Stefani Silva dos SANTOS ${ }^{3}$

Resumo: Este trabalho tenciona analisar brevemente o desempenho de dois Sistemas de Memórias de Tradução (SMTs) com armazenagem em nuvem, a saber: SmartCat e Wordfast Anywhere, no que diz respeito ao processo tradutório (português $\leftrightarrow$ inglês) de termos simples e complexos relacionados ao campo do comércio e exportação de produtos cítricos brasileiros. Valemo-nos, para tanto, de teorias voltadas à Tradução assistida por computador (MENDIBIL, 1995; WEININGER, 2004; STUPIELLO, 2011, 2015), às Memórias de Tradução (RIECHE, 2004, LIMA et al., 2017) e da Tradução técnico-terminológica (VILLELA, 2016; POLCHLOPEK; AIO, 2009; BARROS, 2004). Buscamos verificar alguns aspectos pertinentes ao processo tradutório da linguagem de especialidade presente em Notas Fiscais de Importação e Exportação, entre outros documentos, por meio dos SMTs a fim de investigar as aproximações e distanciamentos no tocante à tal terminologia. Os resultados apontaram para exemplos como "Secretaria de Agricultura e Abastecimento e Coordenadoria de Defesa Agropecuária" / The Secretariat of Agriculture and Supply Department of Agriculture Defense/ Department of Agriculture and Food Suplly Coordination of Agricultural Defense, o que nos leva a salientar a necessidade de enaltecer o papel do tradutor como mediador e condutor de escolhas efetivas dentro das plataformas de MTs.

Palavras-chave: Tradução técnica. Tradução assistida por computador. Terminologias da Exportação e Importação de Cítricos.
Abstract: This work intends briefly to analyze the performance of two Translation Memory Systems (TMSs) which has cloud storage service, namely: SmartCat and Wordfast Anywhere, considering the translational process (Portuguese $\leftrightarrow$ English) for the specific simple and compound terms related to Brazilian trade-export of citrus. We observe theories about Computer-assisted translation (MENDIBIL, 1995; WEININGER, 2004; STUPIELLO, 2011, 2015), Translation Memories (RIECHE, 200, LIMA et al., 2017) and Technical-terminological translation (VILLELA, 2016; POLCHLOPEK; AIO, 2009; BARROS, 2004). We inserted Import and Export Invoices, among other documents, in the TMSs, and by the verification of terms used by authorities in the processes of food exportation and their respective translations. The results pointed to examples of deviation from the language of specialty, as in the case of "Secretaria de Agricultura e Abastecimento e Coordenadoria de Defesa Agropecuária" translated as The Secretariat of Agriculture and Supply Department of Agriculture Defense and as Department of Agriculture and Food Suplly Coordination of Agricultural Defense by SmartCat and Wordfast respectively. This fact leads us to emphasize the role of the translator as a mediator and a driver of effective choices within TM platforms.

Keywords: Technical translation. Computer-assisted translation. Terminology of Export and Import for Citrus.

1 Universidade Estadual Paulista "Júlio de Mesquita Filho" (UNESP), São José do Rio Preto, São Paulo, Brasil; talitasrp82@gmail.com; https://orcid.org/0000-0003-3324-9593

2 União das Faculdades dos Grandes Lagos (UNILAGO), São José do Rio Preto, São Paulo, Brasil; ionedelima30@hotmail.com; http://orcid.org/0000-0003-4976-8945

3 União das Faculdades dos Grandes Lagos (UNILAGO), São José do Rio Preto, São Paulo, Brasil; stefanisantosnh@gmail.com; http://orcid.org/0000-0002-6641-0008 
- Smartcat e Wordfast Anywhere: sistemas de memórias de tradução e a documentação na área agrícola exportadora de limão

\section{Introdução ${ }^{4}$}

Atualmente, a Tradução evoluiu, tornando-se uma das principais atividades nos ramos empresariais, dada a globalização e a abertura de mercado que vivemos desde os anos 2000. Assim, podemos entendê-la como aliada na evolução da tecnologia do comércio internacional, inclusive da comercialização de frutas cítricas, permitindo que se obtenha acesso à informação em qualquer idioma e que se promovam contratos e vínculos entre países que antes não realizavam negócios.

Sendo assim, o processo tradutório assume caráter de ferramenta e apresenta a necessidade de se adequar a um sistema capitalista bastante dinâmico que exige do tradutor destreza, rapidez e eficiência, levando-o a buscar por instrumentos práticos que facilitem a sua atividade, os quais, nas últimas duas décadas, ganharam força com a disseminação da internet, bem como dos meios eletrônicos, como softwares e aplicativos digitais (STUPIELLO, 2011, 2015).

Nesse contexto, os Sistemas de Memórias de Tradução (doravante SMTs) representaram um importante avanço para adequar um processo que é, muitas vezes, linguístico e cultural, a uma demanda de agilidade e de competências que, em outra situação, levaria mais tempo para ser efetuada. Contudo, apesar de extremamente relevante ao papel do tradutor dentro do campo mercadológico, esses sistemas podem apresentar algumas limitações nos processos tradutórios que envolvem as mais diversas linguagens de especialidade e terminologias.

Dessa forma, propomo-nos a analisar as diferentes possibilidades de tradução para termos simples e compostos presentes em textos especializados na área comercialagrícola exportadora de limão na direção português $\leftrightarrow$ inglês apresentadas por dois SMTs, cujo armazenamento é realizado no formato de nuvens, a saber: SmartCat e WordFast Anywhere.

Tencionamos verificar como tais sistematizações podem auxiliar o trabalho do tradutor, por meio de comparações entre traduções dos mesmos textos realizadas por essas duas ferramentas, a fim de procurar comprovar o papel fundamental de tais instrumentos e apontar, possivelmente, aquela que parece exercer uma função mais prática e eficiente do processo tradutório.

4 Tratou-se de um trabalho inicial produzido pelas autoras dentro da disciplina de Prática de Tradução III de um curso de Bacharelado em Letras com Habilitação em Tradução. 
Assim, este trabalho concentra-se em investigar as distintas opções de cada SMT pesquisado, refletindo sobre as conexões entre aproximações e distanciamentos dos textos de partida (TPs) e dos textos de chegada (TCs), bem como verificando as mudanças e aprimoramentos e mostrando o papel do tradutor profissional na manipulação de programas de Memória de Tradução.

\section{Estudos da Tradução: Panoramas teóricos e ferramentas}

Os Estudos da Tradução contemplam aspectos das ciências linguísticas que observam a tradução tanto como um objeto dentro de perspectivas já reconhecidas, como o Estruturalismo, o Funcionalismo e o Descritivismo. Atualmente, contudo, traduzir deixou de ser apenas um fenômeno analisável sob distintas óticas teóricas para se tornar um campo independente de pesquisa. Sendo assim, a transferência de um texto de uma língua fonte (LF) para uma língua meta (LM) passa a constituir um estudo científico importante dedicado à compreensão dos constructos de correspondência objetiva entre mensagens emitidas em diferentes idiomas (MUNDAY, 2004).

Shuttleworth e Cowie, no Dictionary of Translation Studies (1997, p. 184), definem tradução como um processo ou um produto e identificam suas subcategorias, tais como: Tradução Literária, Técnica, Legendagem e Automática, etc. Entretanto, salientam que o termo ainda se refere, em muitos casos, apenas à transferência de textos escritos, embora possa abarcar o conceito de interpretação (SHUTTLEWORTH; COWIE, 1997, p. 184).

Conforme Munday (2004, p. 7), podemos entender Tradução como:

1. O processo de transferência de um texto escrito a partir de LF para uma LM, conduzido por um tradutor ou por tradutores, em um determinado contexto sociocultural.

2. O escrito ou produto, o texto meta, que resulta desse processo e cujas funções se relacionam com o contexto sociocultural.

3. Os fenômenos cognitivos, linguísticos, visuais, culturais e ideológicos que são parte integrante de 1 e 2 .

Assim, entendemos, em nosso trabalho, que a Tradução é a transmissão de mensagens que devem significar aos potenciais leitores inseridos nos mais variados contextos. Além disso, salientamos que os fenômenos cognitivos, apontados pelo autor, caracterizam a atividade tradutória como um fator delimitado pela ação humana. 
- Smartcat e Wordfast Anywhere: sistemas de memórias de tradução e a documentação na área agrícola exportadora de limão

Por conseguinte, a Tradução envolve ideologias, simbologias e escolhas socioculturais, as quais, graças à globalização e ao avanço tecnológico, contam com ferramentas desenvolvidas para auxiliar e agilizar os processos decisórios, dentre elas destacamos a Tradução Automática ou Machine Translation, bem como os SMTs que podem estar a ela associados.

\section{Tradução Automática ou Machine Translation}

O conceito de Machine Translation ou Tradução Automática (TA) refere-se à Tradução realizada com base nas associações ordenadas por uma máquina ou à atividade efetuada total ou parcialmente por um computador (JAKOBSON, 2008). As TAs trazem consigo a proposição de que, ao serem utilizadas, não é necessária a presença do tradutor; entretanto, notamos que os seres humanos ainda são mais hábeis na análise e interpretação da linguagem natural que até mesmo as mais sofisticadas máquinas (STUPIELLO, 2015).

Sendo assim, a TA é um sistema de tradução interlingual (JAKOBSON, 2008) que compreende a análise das frases de um TP, elencando as características morfológicas e sintáticas a serem traduzidas e realizando uma primeira transferência para o TC com uma linguagem intermediária, a qual se utiliza de frases previamente existentes (MENDIBIL, 1995).

Um Sistema de Tradução Automática (STA) compreende apenas características morfológicas, sintáticas (e parcialmente semânticas) das orações, e realiza traduções estruturais, não analisando qualquer elemento extralinguístico ou as particularidades de cada língua. Utiliza aparato tradutório, como corpora, que são baseados, em princípio, em probabilidades do uso de sentenças regulares; para uma série de "palavras-fonte" que funcionam como "entradas", gerando, pelo menos, duas "hipóteses-alvo", cada uma incluindo uma série de novas "palavras-alvo" na LM (MENDIBIL, 1995).

A entrada no primeiro idioma é caracterizada com base em uma ou mais "palavras-fonte" e a frase correspondente no segundo idioma é emitida. Essa abordagem de segmentos permite efetivamente uma tradução rápida e precisa, uma vez que as ocorrências, na segunda língua, são preparadas com antecedência e eventualmente mantidas. Não há, pois, má tradução ou má construção gramatical, visto que, com a escolha adequada, é possível caracterizar um grande número de frases relativamente longas e complexas com apenas algumas "palavras-fonte".

Quando pensamos em um STA, devemos ter em mente que ele compreende meios de absorção da primeira língua e possíveis formas de tradução para a segunda 
língua. Desse modo, a TA envolve os dois idiomas e insere o que é considerado a melhor tradução por meio do conjunto de palavras de maior ocorrência nas combinatórias, produzindo sentidos da principal mensagem inserida pelo usuário.

\section{Memórias de Tradução ou SMTs}

As MTs são ferramentas que acumulam seguimentos, tais como, frases, termos ou fragmentos textuais, para amparar o tradutor em seu trabalho, pois foram desenvolvidas para o ganho de tempo e melhoria na qualidade tradutória. Conforme afirma Rieche (2004, p. 103),

[...] os sistemas de memórias de tradução surgiram prometendo ganho de produtividade, maior consistência e economia, uma vez que são banco de dados que armazenam traduções para reaproveitamento posterior com base em graus de equivalência propostos pelo sistema.

Desse modo, as MTs funcionam como um sistema de armazenamento de informações linguísticas que podem ser reutilizadas em outras traduções, possibilitando o ganho de tempo, bem como novas possibilidades de composição textual. Referir-se a este rendimento, contudo, não significa, necessariamente, falar sobre qualidade. Sendo assim, o profissional da área da Tradução entende que as MTs não realizarão um trabalho independente, já que uma máquina traduz apenas os elementos de ordem estrutural, e não vão além desses aspectos morfológicos do idioma.

Rieche (2004, p. 120) sugere ainda que

Uma das maneiras possíveis de evitar que a memória apresente erros é fazer toda e qualquer alteração sugerida pelo processo de controle da qualidade nos respectivos arquivos sempre utilizando o sistema de memória para garantir que a alteração fique definitivamente armazenada.

Muitos profissionais corrigem os erros de tradução no próprio texto e não na memória, gerando, assim, um aumento de possíveis produções equivocadas. Estando consciente de que erros fazem parte destes sistemas, o especialista da área deve corrigilos, portanto, na memória. A revisão para o tradutor que trabalhar com STAs é a base para um resultado final de qualidade.

Esse processo de correção e inspeção deve atentar-se para aspectos como terminologia, ortografia, concordância e outros pontos gramaticais, para que uma tradução 
- Smartcat e Wordfast Anywhere: sistemas de memórias de tradução e a documentação na área agrícola exportadora de limão

seja de qualidade e o profissional possa reutilizar o banco de dados em outras traduções, uma vez que foi revisado e os segmentos tornaram-se compatíveis para elas.

\section{Smartcat e Wordfast Anywhere: breves considerações}

SmartCat e Wordfast Anywhere são plataformas digitais que prestam auxílio ágil aos tradutores que necessitam concluir trabalhos em prazos cada vez menores. Trata-se de ferramentas criadas recentemente que sempre recebem atualizações, dando plena liberdade ao profissional que elabora seu produto. Conforme Lima et al. (2017, p. 145), no SmartCat:

[...] é possível ter um perfil pessoal ou um corporativo, sendo que neste há a opção de edição colaborativa dos projetos de tradução, o que significa a possibilidade de divisão das tarefas de tradução, edição e revisão entre múltiplas pessoas [...] o usuário encontra seus projetos dispostos em lista para serem visualizados e editados.

Oferece ainda maior facilidade de visualização para a tradução que está sendo feita, pois é organizada de forma que alinha o TP ao TC, possibilitando comparações de ambas as partes e edição rápida, caso necessário. Lima et al. (2017, p. 145) descrevem que na:

[...] interface de edição, o texto se encontra em segmentos paralelos e alinhados dispostos lado a lado com o texto fonte à esquerda e o espaço para a inserção da tradução que formará o texto de chegada à direita, sendo que o texto é segmentado nos pontos finais dos períodos. Essa forma de organização do texto a ser traduzido contribui para ergonomia do ambiente de trabalho, ao posicionar o espaço para a inserção da tradução do segmento ao lado do texto fonte, tornando desnecessária uma maior movimentação dos olhos [...]

Esta plataforma também dispõe de MTs em formato de tmx, que oferecem diversas possibilidades de tradução. Essas memórias estão aptas a conceder traduções instantâneas pelo fato de estarem inteiramente ligadas a traduções geradas precedentemente, alimentando-se, também, de criações de clientes anteriores que compartilharam as suas informações.

O Wordfast Anywhere é um sistema temporário variável e adaptativo em sua estrutura e em sua abordagem para desenvolver novas soluções às traduções. Sua primeira 
versão foi desenvolvida em 1999; na época, havia apenas alguns pacotes de software de MTs disponíveis, com preços exorbitantes, e sua ideia era desenvolver um pacote Powerto-the-people TM a um preço acessível.

\section{A Tradução Técnico-Terminológica}

Como já mencionamos nos tópicos anteriores, a utilização de SMTs aumentou devido à rápida globalização, aos avanços tecnológicos e aos princípios vinculados à indústria de localização, indústria esta que, de acordo com Villela (2016, p. 220), se refere:

[...] ao tipo de tradução técnica de material textual que acompanha os softwares [...] e produtos (manual do usuário, brochuras, sites de divulgação, catálogos etc.) destinados ao público de um país diferente daquele que foi criado (cf. PYM, 2005). O termo também se aplica à tradução de demais adaptações relacionadas à indústria de jogos eletrônicos.

Desse modo, a tradução técnica trabalha com materiais especializados, termos técnicos, tipologias etc. Por ser muito pouco reconhecida ainda, os leigos na área tradutória acreditam que a tradução técnica é apenas o estudo de uma terminologia de determinada área ou assunto, porém a tradução técnica vai muito além de uma terminologia, é a reconstrução de um texto cheio de vocabulário especializado de áreas comerciais e informativas de uma cultura para outra (VILLELA, 2016).

Adentrando os estudos sobre tradução técnica podemos notar, segundo Polchlopek e Aio (2009, p. 105) que:

[a] [tradução] [técnica] é de cunho empírico, não-conceitual, voltada às características estilísticas do texto, dentre as quais observam-se o predomínio de: tempo presente com a função de atingir a objetividade, o factual; uso de asserções, frases curtas e orações simples; pretensão a uma ausência de ambiguidade; pouco uso de adjetivação valorativa; emprego da voz passiva e auxiliares modais, parágrafos curtos e itemizados; dados estilísticos; normalizações (substantivos e adjetivos derivados de verbos); conclusões parciais para cada item abordado, além é claro, da terminologia técnica.

Desta forma, o texto técnico ancora-se em terminologias, pelo fato de a linguagem ser mais específica do que em outros tipos textuais, tipologias e documentos comerciais e informativos (CAVACO-CRUZ, 2012). A sua definição não comporta apenas a parte 
- Smartcat e Wordfast Anywhere: sistemas de memórias de tradução e a documentação na área agrícola exportadora de limão

gramaticalmente estabelecida, e sim um conjunto de itens dentro de um processo, tais como, tempo, linguagem, estrutura, público-alvo, finalidade e cultura.

Vale aqui definir termo como sendo, na acepção do Dicionário de Linguística (DUBOIS et al., 1979, p. 614), um emprego monossêmico de uma unidade lexical dentro do domínio de uma área de especialidade. Barros (2004, p. 42) esclarece que

[...] termo é um vocábulo, uma vez que é um modelo de realização lexical no texto. Seu caráter de termo se dá pelo fato de que designa um conceito específico de um domínio de especialidade. O conjunto terminológico presente nesse texto constitui, na verdade, um subconjunto do conjunto vocabular do mesmo. Assim, um termo é também um vocábulo, além de ser uma palavra.

Na Terminologia (BARROS, 2004), unidades lexicais expandidas que designam um conceito específico dentro de um contexto dado e de um domínio de especialidade passam a apresentar um caráter de termos complexos.

A autora aponta que um termo simples pode ser compreendido como "construído de um só radical, com ou sem afixos" (ISO 1087, 1990, p. 7), ao passo que um termo complexo é "constituído de dois ou mais radicais, aos quais se podem acrescentar outros elementos" (ISO 1087, 1990, p. 7). No que concerne aos termos compostos, Barros (2004, p. 100) considera que:

[...] também são unidades lexicais formadas por dois ou mais radicais. Distinguem-se, no entanto, dostermos complexos pelo alto grau de lexicalização e pelo conjunto de morfemas lexicais e/ou gramaticais que os constitui, em situação de não-autonomia representada graficamente pela utilização do hífen. [...] Cumpre ressaltar que consideramos as unidades lexicais complexas por aglutinação (como fidalgo, embora, etc.) e pela justaposição sem hífen de dois ou mais radicais como termos simples.

Essa particularidade faz com que termos simples e compostos sejam utilizados em todas as línguas em virtude da junção de aspectos referentes ao contexto de comunicação e de aspectos da experiência.

É, no entanto, na tradução técnica que é requerido o maior uso de termos, uma vez que geralmente está relacionada a manuais, textos instrutivos ou guias gerais. Este procedimento é voltado para a utilização especializada de materiais e linguagens que já possuem conceitos e ideias concretas direcionadas por algum assunto. Para isso, é necessário um bom conhecimento do profissional que domine determinada terminologia. 
Ao estudarmos a tradução técnica, interagimos com diversos conceitos indispensáveis, e encontramos, a partir das diferenças da linguagem, o redirecionamento aos elementos técnicos de determinado assunto. Azenha (1996, p. 142) afirma haver distinções entre "comum" e "técnico" e que "o processo de produção dos textos técnicos está sujeito a variáveis que são diferentes em grau, mas não em essência, das que influenciam a produção e a recepção de qualquer tipo de texto".

\section{Material e Método}

Para o desenvolvimento desta investigação, construímos um corpus pautado em documentos relacionados ao comércio e exportação de produtos cítricos em Português Brasileiro, os quais estão elencados a seguir:

1) Notas fiscais de importação e exportação;

2) Permissão de trânsito para vegetais (PTV);

3) Certificado Fitossanitário de origem consolidado (CFOC);

4) Certificado Fitossanitário de Origem (CFO); e

5) Certificado Fitossanitário.

A seguir, compilamos um corpus comparável com documentos também pertinentes à exportação de hortifrútis brasileiros, os quais foram originalmente escritos em Inglês ${ }^{5}$ como é o caso dos:

1) Final Report of an Audit Carried Out in Brazil from 07 to 18 November 2011;

2) Mission of the FYO in Brazil From 9 to 17 of August 2004;

3) International Standards for Phytosanitary Measures Ispm 12 Phytosanitary Certificates (2011); e

4) Completion of a Phytosanitary Certificate User Guide by Australian Government.

5 Há documentos de diferentes países de língua inglesa, não havendo qualquer especificação em nosso trabalho acerca de diferenciações neste âmbito. 
- Smartcat e Wordfast Anywhere: sistemas de memórias de tradução e a documentação na área agrícola exportadora de limão

Além disso, valemo-nos de dez artigos científicos especializados em comércio internacional de produtos agrícolas brasileiros e seus derivados originalmente em inglês, bem como de dados dos seguintes órgãos de regulamentação agrária: Secretary of Agriculture and Cattle Rising Defense (Brazil); Food and Agriculture Organization of the United Nations (FAO) e HF Rural ${ }^{6}$.

Inserimos os documentos em LF nos dois SMTs com armazenamento em nuvem que nos dispusemos a verificar e selecionamos dez termos simples e complexos ${ }^{7}$ e verificamos as possibilidades de tradução fornecidas pelas memórias, procurando aproximações e distanciamentos com relação aos dados do corpus comparável, os quais corroboram a circulação das frutas (cítricas) entre produtores e comerciantes-exportadores do Brasil.

\section{Análise dos dados}

Conforme visto na seção Material e Método, os textos da área de exportação de limão em LF foram inseridos nos SMTs a serem estudados. Cada texto foi traduzido automaticamente, e obtivemos, assim, alguns dos principais resultados analisados no decorrer deste tópico.

Dessa maneira, apresentamos, a seguir, os termos simples e complexos levantados nos documentos selecionados, entre os quais selecionamos dez exemplos específicos, os quais subcategorizamos conforme seus usos, a saber: 1) Normas/Legislações agrárias; 2) Transporte e Segurança e 3) Produtos.

\section{Normas/ Legislação Agrária}

As normas que delimitam a venda de produtos agrícolas brasileiros aos mercados internacionais são gerenciadas pela Secretaria da Agricultura, a qual exige documentos específicos, a fim de garantir o compromisso com a qualidade das frutas, de modo que gerencia propriedades rurais e empresas exportadoras, com o intuito de evitar a propagação de pragas e pesticidas e de consolidar o mercado.

Dessa forma, a venda de frutas cítricas para outros países está vinculada ao correto preenchimento dos seguintes documentos: Certificado Fitossanitário de Origem (CFO), Certificado Fitossanitário, Certificado Fitossanitário de Origem Consolidado (CFOC) e Permissão de Trânsitos para Vegetais. Apresentamos, a seguir, a lista com exemplos desses termos e suas possíveis traduções em LM.

6 Site desenvolvido com intuito de expor a regulamentação das vendas de frutas e legumes no Brasil e no exterior.

7 Os termos compostos não foram abordados em nossa investigação. 
Quadro 1. Tradução dos termos complexos de Legislação Agrária presentes nos documentos selecionados

\begin{tabular}{|c|c|c|c|}
\hline Termo & SmartCat & $\begin{array}{l}\text { Wordfast } \\
\text { Anywhere }\end{array}$ & $\begin{array}{l}\text { Tradução oficial } \\
\text { com base no corpus } \\
\text { comparável }\end{array}$ \\
\hline $\begin{array}{c}\text { Secretaria de } \\
\text { Agricultura e } \\
\text { Abastecimento e } \\
\text { Coordenadoria de } \\
\text { Defesa Agropecuária }\end{array}$ & $\begin{array}{l}\text { The Secretariat of } \\
\text { Agriculture and } \\
\text { Supply Department } \\
\text { of Agriculture } \\
\text { Defense }\end{array}$ & $\begin{array}{c}\text { Department of } \\
\text { Agriculture and Food } \\
\text { Suplly Coordination } \\
\text { of Agricultural } \\
\text { Defense }\end{array}$ & $\begin{array}{c}\text { Secretary of Agriculture } \\
\text { and Cattle Rising } \\
\text { Defense }\end{array}$ \\
\hline $\begin{array}{l}\text { Certificado } \\
\text { Fitossanitário de } \\
\text { Origem (CFO) }\end{array}$ & $\begin{array}{c}\text { Phytosanitary } \\
\text { Certificate of Origin }\end{array}$ & $\begin{array}{c}\text { Phytosanitary } \\
\text { Certificate of Origin }\end{array}$ & $\begin{array}{c}\text { Origin Phytosanitary } \\
\text { Certificate/ } \\
\text { Phytosanitary Certificate } \\
\text { of Origin }\end{array}$ \\
\hline $\begin{array}{c}\text { Certificado } \\
\text { Fitossanitário (CF) }\end{array}$ & $\begin{array}{l}\text { Phytosanitary } \\
\text { Certificate }\end{array}$ & $\begin{array}{l}\text { Phytosanitary } \\
\text { Certificate }\end{array}$ & Phytosanitary Certificate \\
\hline $\begin{array}{c}\text { Permissão de Trânsitos } \\
\text { para Vegetais }\end{array}$ & $\begin{array}{l}\text { Permission of Transit } \\
\quad \text { for Vegetable }\end{array}$ & $\begin{array}{c}\text { Permission of Transit } \\
\text { Plant }\end{array}$ & $\begin{array}{l}\text { Vegetables Traffic } \\
\text { Permission }\end{array}$ \\
\hline
\end{tabular}

Fonte: Elaboração própria

Nos exemplos selecionados, notamos que a tecnicidade do texto não está apenas amparada no terminológico, mas atrelada aos fatores legais e à maneira como cada país estabelece suas regras de importação e exportação, bem como cria suas agências reguladoras.

Sendo assim, é preciso que o processo tradutório estabeleça uma imbrincada relação não somente com os parâmetros presentes no TP, como também com o reconhecimento de elementos externos ao próprio texto. É interessante pontuar, aqui, que quando verificamos uma documentação, o seu caráter de legalidade eleva o patamar de padronização dos termos, de forma que os sistemas utilizados não estão devidamente amparados com MTs para realizar traduções sistematicamente técnicas, sendo a intervenção do tradutor requerida na elaboração dos produtos finais, para que estejam adequados à normatização do mercado.

No caso da "Secretaria de Agricultura e Abastecimento e Coordenadoria de Defesa Agropecuária", é interessante observar que os SMTs apresentam as seguintes soluções: The Secretariat of Agriculture and Supply Department of Agriculture Defense e Department of Agriculture and Food Suplly Coordination of Agricultural Defense. Contudo, o Ministério da Agricultura e Pecuária (Brasil) tem sua nomenclatura convencionalizada como Secretary of Agriculture and Cattle Raising Defense, conforme as bases presentes no próprio Ministério. 
- Smartcat e Wordfast Anywhere: sistemas de memórias de tradução e a documentação na área agrícola exportadora de limão

Quadro 2. Exemplo do uso do termo complexo Secretary of Agriculture and Cattle Raising Defense dentro dos documentos consultados como corpus comparável

NORMATIVE INSTRUCTION No. 1, OF JANUARY 11th, 2017 THE SECRETARY OF AGRICULTURE AND CATTLE RAISING DEFENSE, DEPUTY, OF THE MINISTRY OF AGRICULTURE, LIVESTOCK AND SUPPLY, in the use of the attributions granted to him by art. 18, subparagraph II, letter "I", and art. 53, both of Attachment I of Decree no. 8.852, of September 20th, 2016, considering the provisions of Law no. 1.283, of December 18th, 1950, in Decree no. 30.691, of March 29th, 1952, and what is contained in Process no. 21000.021334/2016- 62 ${ }^{8}$

Fonte: Elaboração própria

Cremos que a melhor opção a ser utilizada é a que o Ministério oferece, uma vez que se tornou um termo compartilhado entre os usuários de seus serviços. Assim, refletindo sobre como as SMTs traduziram o termo, notamos que ocorre um distanciamento do processo tradutório comumente aceito, uma vez que não há amparo pelos elementos culturais e sociais e mantêm-se os significados independentes, mostrando a importância do tradutor diante de termos técnicos, os quais sistemas altamente tecnológicos ainda não são aptos a traduzir.

Outro exemplo apresentado no quadro 1 é "Certificado Fitossanitário de Origem", traduzido como Phytosanitary Certificate of Origin pelos SMTs, corroborando as traduções apresentadas pelos documentos utilizados para apoio, as quais apresentam a seguinte opção Phytosanitary Certificate of Origin, o que caracterizaria uma aproximação e uma possibilidade de tradução plausível, uma vez que seu uso é frequente nos textos relacionados aos cuidados das frutas, conforme os exemplos apresentados no quadro 3.

8 Exemplo retirado do website do Ministério da Agricultura no endereço: http://www.agricultura.gov.br. 
Quadro 3. Exemplo do uso do termo complexo Phytosanitary Certificate of Origin nos textos que compõem o corpus comparável

This Norm, consisting of 16 articles, provides for the Phytosanitary Certificate of Origin (CFO), Phytosanitary Certificate of Verified Origin (CFOC) and Vegetable Transit Permit (PTV) in electronic format. It aims to implement, in every State of Espírito Santo, the remote form of requesting the Electronic Vegetable Transit Permit, called e-PTV, for the interstate transit of vegetables and their parts. The control and issuance of CFO, CFOC and PTV will be carried out through SIMLAM or its success.

Fonte: Elaboração própria

Observando o exemplo, é perceptível que, embora os sistemas não apresentem MTs para termos da legislação, a sequência morfológica e sintática favorece a tradução da sequência do termo e permite encontrar opções condizentes com os textos documentais que circulam entre os órgãos reguladores.

No entanto, é possível verificar que não existe consenso quanto ao uso do termo, uma vez que também foram encontrados exemplos em que a tradução Origin Phytosanitary Certificate ocorreu, conforme exemplo a seguir:

Quadro 4. Exemplo do uso do termo complexo Origin Phytosanitary Certificate nos textos que compõem o corpus comparável

In relation to the inspections and control accomplished by the federal, state services and for private $f$ technicians that form the export process that contemplates the Brasilian Origin Phytosanitary Certification System, composed by the next documents: Origin Phytosanitary Certificate - CFO; Phytosanitary Certificate of Consolidated Origin - CFOC; vegetables traffic permission - PTV; Phytosanitary Certificate - CF.

Fonte: Elaboração própria (2018)

Não se trata, por conseguinte, somente de uma adequação terminológica, mas sim da necessidade de se reconhecer os limites dentro da linguagem do sistema de regulamentação da compra e venda de cítricos, o qual não apresenta uma linguagem bem esquadrinhada mesmo entre seus usuários.

O papel do tradutor, neste âmbito, perpassa o das escolhas efetivas dentro de um conjunto de termos previamente definidos e ganha a proposição de padronizar a linguagem de especialidade, utilizando, para isso, entre outros instrumentos, as ferramentas dos SMTs, visto que, uma vez alterado o item em uso ou sua sequência dentro da memória, há uma convencionalidade por se repetir essa opção. 
- Smartcat e Wordfast Anywhere: sistemas de memórias de tradução e a documentação na área agrícola exportadora de limão

\section{Transporte e Segurança}

Em segundo lugar, analisamos a terminologia que se refere ao transporte e à segurança, uma vez que, para a fruta chegar de um país ao outro, é necessário passar por um processo de deslocamento que é regulamentado por procedimentos de segurança que mantêm a qualidade do produto.

Dessa maneira fraudes são evitadas, bem como o país receptor da fruta recebe informações acerca do transporte e da manutenção. Escolhemos os itens a seguir para demonstrar como os programas traduziram e quais as possíveis atuações dos tradutores.

Quadro 5. Tradução dos termos de Transporte e segurança

\begin{tabular}{|c|c|c|c|}
\hline Termo & SmartCat & Wordfast Anywhere & Tradução Oficial \\
\hline Container & Contanier & Container & Container \\
\hline Lacre & Seal & Seal & Seal \\
\hline Termógrafo & Thermographic & Thermographic & Thermographic \\
\hline
\end{tabular}

Fonte: Elaboração própria

Ao tratarmos os itens mostrados no quadro, podemos perceber que os termos também são de alta tecnicidade, uma vez que representam fatores e elementos essenciais dentro dos sistemas de escoamento de produtos e materiais no Brasil.

Quando nos referimos ao transporte, temos que nos ater à segurança do produto, no caso de nossa pesquisa, do limão. Nesse sentido, as cargas devem ser lacradas e, nas Notas Fiscais, tal informação deve estar explicitada, como em: "lacre HLD4525895", exemplo traduzido pelos programas como seal HLD4525895.

No Cambridge Online Dictionary (2018, tradução nossa ${ }^{9}$ ), encontramos, para a palavra seal, a seguinte definição "um pedaço fino de material como papel ou plástico que tem que ser quebrado para abrir um recipiente". Identificamos que o lacre, seal, é o que deve ser quebrado, no caso, para abrir um container para ver a fruta. Devese quebrar o lacre, e isso acontece, normalmente, no porto exportador, lugar no qual todos os documentos citados em Normas e Legislação dos produtos são exigidos para a conferência.

Notamos que, por ser um termo simples, os SMTs são capazes de recuperar fragmentos de traduções já utilizados. Além disso, podem estar presentes em textos de outras naturezas, embora apareçam com frequência no corpus comparável.

9 No original: "a thin piece of material such as paper or plastic that has to be broken to open a container, envelope, etc.". 
Quadro 6. Exemplo do uso do termo seal nos textos que compõem o corpus comparável

The Phytosanitary certificates for citrus exported to EU, with exception of Tahiti lime, are issued in the pack house by the Federal inspectors, after the consignment has been already loaded on the ship. The reason for this, as stated by the Federal inspector, is to have the correct seal number indicated on the PC, since Customs inspection are often carried out at the port, after which the original seal is replaced.

Fonte: Elaboração própria

O mesmo se dá com o exemplo de Contanier, considerando que a utilização de termos já definidos em documentos comerciais é bastante reticente e que tanto as Notas Fiscais quando as PTVs apresentam a mesma designação Container - HLXU 878.493-4.

Quadro 7. Exemplo do uso do termo container nos textos que compõem o corpus comparável

Following the final inspection, the consignment is loaded in the containers and sealed at the pack house. The PC is issued by Federal inspectors in the pack house but at a later stage when the consignment is already loaded on the ship.

Fonte: Elaboração própria

Parece-nos haver aqui a regularidade que não existe na terminologia relacionada aos documentos e órgãos expedidores, decorrente, talvez, do rearranjo do termo em língua portuguesa, "contêiner" ou "contentor". No entanto, notamos que, apesar de haver uma nomenclatura para o equipamento de transporte no Brasil, seu uso no corpus em estudo se dá por meio do empréstimo da língua inglesa. Assim, o padrão se perde não na tradução, mas sim na própria composição documental brasileira que não se utiliza da possibilidade oferecida pelo conjunto léxico do português.

Vale ressaltar, ainda que, em LF, um contêiner é destinado ao acondicionamento e transporte de cargas, normalmente em navios e trens. São denominados "cofres de carga", pois, conforme mencionado, são dotados de dispositivos de segurança previstos pela legislação nacional e também por convenções internacionais.

\section{Produto}

Por fim, atentamo-nos ao produto, ressaltando que o limão é uma fruta internacionalmente conhecida e que o Brasil é reconhecido mundialmente por ser um de seus maiores exportadores. Além disso, localiza-se no município de Itajobi (distrito 
- Smartcat e Wordfast Anywhere: sistemas de memórias de tradução e a documentação na área agrícola exportadora de limão

Nova Cardoso) a maior produção da fruta no país, sendo este município responsável por grande parte da exportação realizada (GLOBO, 2018).

Abaixo, apresentamos o quadro 8, com exemplos de produtos derivados da citrus latifólia, espécie de fruta cítrica conhecida pelo nome de "Lima Ácida Tahiti" ou "Limão Tahiti", sendo que este último pode ser classificado em três tipos: graúdo, miúdo e a moagem que se referem ao tamanhos e qualidades das frutas que são exportadas e estão descritas nos documentos trabalhados em nossa investigação.

Quadro 8. Tradução dos termos que nomeiam os produtos exportados

\begin{tabular}{|c|c|c|c|}
\hline Termo & SmartCat & Wordfast Anywhere & Tradução Oficial \\
\hline Lima acida tahiti & Acid File Tahiti & Acid File Thaiti & Tahiti Acid File \\
\hline Limão tahiti graúdo & Lemon Tahiti Big & Tahiti Lime Big & Limão Tahiti graúdo \\
\hline Caipirinha & Caipirinha & Caipirinha & Caipirinha \\
\hline
\end{tabular}

Fonte: Elaboração própria

Podemos observar, no primeiro exemplo, que os dois SMTs traduzem termos da mesma maneira. Contudo, quanto à "Lima Ácida Tahiti", há um distanciamento entre as produções dos sistemas estudados, Acid File Tahiti, e a tradução oficializada, Tahiti Acid File, de acordo com a HF RURAL (2018).

Abaixo apresentamos o quadro 9, com um exemplo de trecho em que o termo é utilizado:

Quadro 9. Exemplo do uso do termo complexo Tahiti acid file nos textos que compõem o corpus comparável

A Tahiti acid file offer was controlled in the state of São Paulo in the first half of 2018. This factor, coupled with strong industrial and external demand, sustained the prices of the variety at levels above 2017 (except in March / 18), even at peak harvest.

To the Tahiti acid file, rains in the main producing regions interrupted the harvest of the fruit, raising prices.

Fonte: Elaboração própria

Já no tocante à "caipirinha", os programas mantiveram como opção de tradução caipirinha, levando-nos a considerar que os SMTs não apresentam o termo como parte dos dados de seus armazenamentos ou já apresentavam traduções corroboradas pela indústria exportadora e pelos pesquisadores do uso da cana-de-açúcar. A seguir, o quadro 10 traz alguns exemplos desses usos. 
Quadro 10. Exemplo do uso do termo complexo caipirinha nos textos que compõem o corpus comparável

In Brazil, alcohol made from sugar cane is mixed with lime juice and a little of the cane sugar itself to make caipirinhas - and it's a fine way to get the weekend off to a flying start.

Fonte: Elaboração própria

Podemos compreender as opções de tradução de duas maneiras:

A) Mantém-se o termo por ser específico;

B) Encontram o termo no SMTs, advindo de textos provenientes de outras áreas de especialidade;

É pertinente, então, compreender-se o contexto, uma vez que a "caipirinha" é tipicamente brasileira, e, consequentemente, caraterística do país. Desse modo, trata-se de um termo, embora os programas talvez não o tenham traduzido por reconhecerem que há esse caráter de especificidade. Assim, o tradutor técnico baseia-se em Azenha (1996) quando o autor diz que o texto técnico deve diferir em graus, mas não deve perder sua essência, e, desse modo, mudar o termo alteraria o sentido no TM.

\section{Considerações finais}

A partir dos elementos estudados, foram relatados experimentos realizados com os dois SMTs, o SmartCat e WordFast Anywhere. Dessa forma, mostramos, por meio de documentos utilizados na área agrícola produtora de limão na direção português $\leftrightarrow$ inglês, a importância da intervenção do profissional da tradução, uma vez que esses programas apresentaram limitações em terminologias específicas, causando distanciamentos entre suas opções e os documentos que oficialmente circulam no universo da exportação da fruta.

Diante disso, notamos que, conforme essas ferramentas permitem o ganho de tempo, também conduzem possíveis desvios terminológicos os quais, ao serem propagados, geram problemas na comunicação entre leitores, e, no caso, entre países e clientes do setor de exportação.

O tradutor faz-se necessário porque é capaz de adequar os afastamentos da linguagem técnica regular produzidos pelos SMTs. Assim, corrige possíveis desvios para que a reutilização de termos se torne real, permitindo o real aumento da produtividade, uma vez que, ao corrigir os erros dentro da memória, a terminologia é salva e propagada corretamente. 
- Smartcat e Wordfast Anywhere: sistemas de memórias de tradução e a documentação na área agrícola exportadora de limão

Em suma, é sabido que os SMTs e o indivíduo que produz a tradução fazem parte da aquisição e da intenção de um trabalho valoroso que seja capaz de transparecer a mensagem principal como produto de teor significativo. Assim, nossa reflexão sobre o objeto estudado é amparada pela hipótese de que, ainda que em posse desses dois sistemas, a análise e geração linguística também devem ser grandes aliados na composição do ofício do tradutor.

\section{Referências}

AZENHA JR, J. Tradução Técnica, Condicionantes Culturais e os Limites da Responsabilidade do Tradutor. Cadernos de Tradução, Florianópolis, v. 1, n. 1, p. 137-149, 1996.

BARROS, L. A. Curso básico de terminologia. São Paulo: EDUSP, 2004.

CAMBRIDGE ONLINE DICTIONARY. Disponível em: https:// dictionary.cambridge.org/ pt/. Acesso em: 20 jul. 2018.

CAVACO-CRUZ, L. Manual práctico e fundamental de tradução técnica. s.l.: Arkonte, 2012.

DUBOIS, J. et al. Diccionario de lingüística. São Paulo: Alianza, 1979.

G1.GLOBO.COM. Disponível em: http://g1.globo.com/sao-paulo/sorocaba-jundiai/ nosso-campo/videos/v/itajobi-se-destaca-pela-producao-e-exportacao-de-limaotaiti/6490925/. Acesso em: 20 jul. 2018.

JAKOBSON, R. Linguística e comunicação. São Paulo: Cultrix, 2008.

LIMA, E. B. A. et al. Smartcat: Programa de Apoio à Tradução Online e Gratuito. Cultura e Tradução, v. 4, n. 1, 2017.

MENDIBIL, G. U. E. D. Automatic interlingual translation system. U.S. Patent n. 5, p. 426-583, 20 jun. 1995.

MUNDAY, J. Introducing translation studies. London and New York: Routledge, 2004. 
POLCHLOPEK, S.; AIO, M. A. Tradução técnica: armadilhas e desafios. Tradução \& Comunicação - Revista Brasileira de Tradutores, Florianópolis, n. 19, p. 101-113, 2009.

RIECHE, A. C. Memórias de tradução: auxílio ou empecilho? 2004. Dissertação (Mestrado em Letras) - Pontifícia Universidade Católica do Rio de Janeiro, Rio de Janeiro, 2004.

SHUTTLEWORTH, M.; COWIE, M. Dictionary of Translation Studies. Manchester: St Jerome, 1997.

STUPIELLO, E. N. A. As práticas de tradução redefinidas pelas relações linguísticas na economia informacional. Trabalhos em linguística aplicada, Campinas, v. 50, n. 2, p. 341-356, dez. 2011.

STUPIELLO, E. N. A. O ensino de sistemas de memórias de tradução na graduação: desafios e perspectivas. Estudos Linguísticos, v. 44, n. 2, p. 884-894, ago. 2015.

VILLELA, A. L. C. Software livre e de código aberto no ensino de Programas de Apoio à Tradução: OmegaT, uma alternativa viável. Texto Livre: Linguagem e Tecnologia, v. 9, n. 2, p. 219-230, 2016.

COMO CITAR ESTE ARTIGO: SERPA, Talita; LIMA, Ione Marina de; SANTOS, Stefani Silva dos. Smartcat e Wordfast Anywhere: sistemas de memórias de tradução e a documentação na área agrícola exportadora de limão. Revista do GEL, v. 17, n. 2, p. 267-285, 2020. Disponível em: https://revistadogel.gel.org.br/

DOI: http://dx.doi.org/10.21165/gel.v17i2.2845

Submetido em: 07/06/2020 | Aceito em: 24/06/2020. 